CERN-TH/2001-242

August 2001

\title{
Direct CP Violation In Radiative $B$ Decays
}

\author{
Tobias Hurth* and Thomas Mannel ${ }^{\dagger}$ \\ ${ }^{*}$ CERN, Theory Division, CH 1211 Geneva 23, Switzerland \\ ${ }^{\dagger}$ Institut für Theoretische Teilchenphysik, Universität Karlsruhe, D-76128 Karlsruhe, Germany
}

\begin{abstract}
We discuss the role of the radiative $B$ decays $B \rightarrow X_{s / d} \gamma$ in our search for new physics focusing on the issue of direct $\mathrm{CP}$ violation. We discuss in some detail a SM prediction for the $\mathrm{CP}$ asymmetries in inclusive $b \rightarrow s / d$ transitions, namely $\left|\Delta \mathcal{B}\left(B \rightarrow X_{s} \gamma\right)+\Delta \mathcal{B}\left(B \rightarrow X_{d} \gamma\right)\right| \sim 1 \cdot 10^{-9}$. Any measured value in significant deviation of this estimate would indicate new sources of $C P$ violation beyond the CKM phase.
\end{abstract}

Invited talks given by T.H. at the IPPP Workshop 'Phenomenology of Physics Beyond the SM', Durham (England), 6-11 May 2001, and at the International Workshop on QCD 'QCD@Work', Martina Franca (Italy), 16-20 June 2001; to appear in the Proceedings.

\section{INRODUCTION}

It is well-known that radiative $B$ decays like $B \rightarrow X_{s / d} \gamma$ play an important role in our search of new physics. These processes test the SM directly on the quantum level and, thus, are particularly sensitive to new physics (for a recent review see [1]). While the direct production of new (supersymmetric) particles is reserved for the planned hadronic machines such as the LHC at CERN, the indirect search of the $B$ factories already implies significant restrictions for the parameter space of supersymmetric models and will thus lead to important clues for the direct search of supersymmetric particles. It is even possible that these rare processes lead to the first evidence of new physics by a significant deviation from the SM prediction, for example in the observables concerning direct $\mathrm{CP}$ violation, although it will then be difficult to identify in this way the new structures in detail. But also in the long run, after new physics has already been discovered, these decays will play an important role in analyzing in greater detail the underlying new dynamics.

One of the main difficulties in examining the observables in $B$ physics is the influence of the strong interaction. As is well known, for matrix elements dominated by long-distance strong interactions there is no adequate quantitative solution available in quantum field theory. The resulting hadronic uncertainties restrict the opportunities in $B$ physics significantly. If new physics does not show up in $B$ physics through large deviations as recent experimental data indicates the focus on theoretically clean variables like inclusive radiative $B$ decays is mandatory. 
Within inclusive decays like $B \rightarrow X_{s / d} \gamma$ the long-distance strong interactions are less important and well under control due to the heavy mass expansion. The decay $B \rightarrow X_{s} \gamma$ was first observed by the CLEO collaboration [2]; these measurements have been refined $[3,4]$ and confirmed by other experiments [5, 6] (see also [7]). The present world average using the present data from BELLE, CLEO and ALEPH is

$$
\mathcal{B}\left(B \rightarrow X_{s} \gamma\right)=(3.22 \pm 0.40) \times 10^{-4}
$$

The theoretical prediction of the Standard Model (SM) up to next-to-leading logarithmic (NLL) precision for the total decay rate of the $B \rightarrow X_{s} \gamma$ mode [8] is well in agreement with the experimental data. The theoretical NLL prediction for the $B \rightarrow X_{s} \gamma$ branching ratio [12] leads to

$$
\mathcal{B}\left(B \rightarrow X_{s} \gamma\right)=(3.32 \pm 0.14 \pm 0.26) \times 10^{-4}
$$

where the first error represents the uncertainty regarding the scale dependences, while the second error is the uncertainty due to the input parameters. In the second error the uncertainty due to the parameter $m_{c} / m_{b}$ is dominant.

This inclusive mode already allows for theoretically clean and rather strong constraints on the parameter space of various extensions of the SM [9, 10, 11]. While many phenomenolgical analyses of the inclusive $B \rightarrow X_{s / d} \gamma$ decays in supersymmetry are retricted to the assumption of minimal flavour violation including only CKM induced flavour change, a model-independent analysis also has to consider the generic new supersymmetric sources of flavour change due to the mixing in the squark mass matrix. In [11] new model-independent bounds on supersymmetric flavour-violating parameters were derived from $B \rightarrow X_{s} \gamma$. The importance of interference effects for the bounds on the parameters in the squark mass matrices within the unconstrained MSSM is explicitly demonstrated. In former analyses no correlations between the different sources of flavour violation were taken into account [10]. The new bounds are in general one order of magnitude weaker than the original bounds on the single off-diagonal element, which was derived in previous work [10] by neglecting any kind of interference effects.

Recently, quark mass effects within the decay $B \rightarrow X_{s} \gamma$ were further analysed [13], in particular the definitions of the quark masses $m_{c}$ and $m_{b}$. The charm quark enters in specific NLL matrix elements (see Fig. 1) where the charm quark mass is dominantly off-shell. Therefore, the authors of [13] argue that the running charm mass should be chosen instead of the pole mass. The latter choice was used in all previous analyses $[14,8,15,16,12]$.

$$
m_{c}^{\text {pole }} / m_{b}^{\text {pole }} \Rightarrow m_{c}^{\overline{\mathrm{MS}}}(\mu) / m_{b}^{\text {pole }}, \mu \in\left[m_{c}, m_{b}\right]
$$

Since these matrix elements start at NLL order only and, thus, the renormalization scheme for $m_{c}$ and $m_{b}$ is an NNLL issue, one should regard this choice as an educated guess of the NNLL corrections. The new choice is guided by the experience gained from many higher-order calculations in perturbation theory. Numerically, the shift from $m_{c}^{\text {pole }} / m_{b}^{\text {pole }}=0.29 \pm 0.02$ to $m_{c}^{\overline{\mathrm{MS}}}(\mu) / m_{b}^{\text {pole }}=0.22 \pm 0.04$ is rather important and leads to a $+11 \%$ shift of the central value of the $B \rightarrow X_{s} \gamma$ branching ratio. With their new 


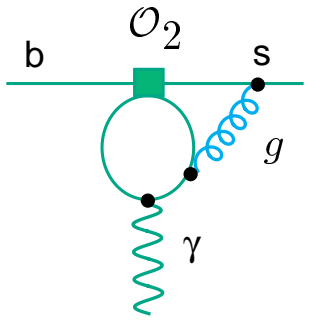

(a)

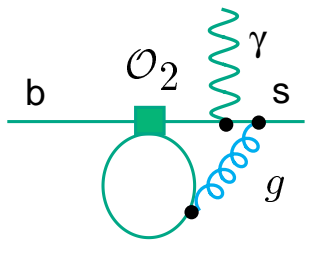

(a)

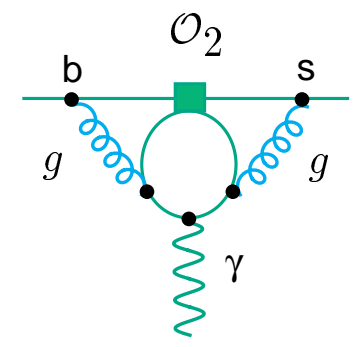

(b)

FIGURE 1. a) Typical diagrams contributing to the matrix element of the operator $\mathrm{O}_{2}$ at the NLL level. b) Typical diagram contributing to the NLL anomalous dimension matrix.

choice of the charm mass their theoretical prediction for the branching ratio is [13]

$$
\mathcal{B}\left(B \rightarrow X_{s} \gamma\right)=(3.73 \pm 0.30) \times 10^{-4},
$$

which induces a small difference between the theoretical and the experimental value.

However, because the choice of the renormalization scheme for $m_{c}$ and $m_{b}$ is a NNLL effect, their important observation should be reinterpreted into a larger error bar in

$m_{c}^{\overline{\mathrm{MS}}}(\mu) / m_{b}^{\text {pole }}$ which includes also the value of $m_{c}^{\text {pole }}$. A more conservative choice would then be $m_{c}^{\overline{\mathrm{MS}}}(\mu) / m_{b}^{\text {pole }}=0.22 \pm 0.07$ which deletes the significance of the perceived discrepancy.

\section{DIRECT CP ASYMMETRY}

The $\mathrm{CP}$ violation in the $B$ system will yield an important independent test of the SM description of $\mathrm{CP}$ violation (see [17]). In particular, detailed measurements of CP asymmetries in rare $B$ decays will be possible in the near future. Theoretical predictions for the normalized $\mathrm{CP}$ asymmetries of the inclusive channels (see $[18,19,20]$ ) within the SM lead to

$$
\begin{gathered}
\alpha_{C P}\left(B \rightarrow X_{s / d} \gamma\right)=\frac{\Gamma\left(\bar{B} \rightarrow X_{s / d} \gamma\right)-\Gamma\left(B \rightarrow X_{\bar{s} / \bar{d} \gamma)}\right.}{\Gamma\left(\bar{B} \rightarrow X_{s / d} \gamma\right)+\Gamma\left(B \rightarrow X_{\bar{s} / \bar{d}} \gamma\right)} \\
\alpha_{C P}\left(B \rightarrow X_{s} \gamma\right) \approx 0.6 \%, \quad \alpha_{C P}\left(B \rightarrow B_{d} \gamma\right) \approx-16 \%
\end{gathered}
$$

when the best-fit values for the CKM parameters [21] are used. An analysis for the leptonic counterparts is presented in [22]. The normalized CP asymmetries may also be calculated for exclusive decays; however, these results are model-dependent. An example of such a calculation may be found in [23].

CLEO has already presented a measurement of the CP asymmetry in inclusive $b \rightarrow s \gamma$ decays, yielding [24]

$$
\alpha_{C P}\left(B \rightarrow X_{s} \gamma\right)=(-0.079 \pm 0.108 \pm 0.022) \times(1.0 \pm 0.030)
$$


which indicates that very large effects are already excluded.

Supersymmetric predictions for the CP asymmetries in $B \rightarrow X_{s / d} \gamma$ depend strongly on what is assumed for the supersymmetry-breaking sector and are, thus, a rather modeldependent issue. The minimal supergravity model cannot account for large $\mathrm{CP}$ asymmetries beyond $2 \%$ because of the constraints coming from the electron and neutron electric dipole moments [25]. However, more general models allow for larger asymmetries, of the order of $10 \%$ or even larger $[26,19]$. Recent studies of the $B \rightarrow X_{d} \gamma$ rate asymmetry in specific models led to asymmetries between $-40 \%$ and $+40 \%$ [28] or $-45 \%$ and $+21 \%$ [27]. In general, CP asymmetries may lead to clean evidence for new physics by a significant deviation from the SM prediction.

In [29] it was explicitly derived, that a bound on the combined asymmetries within the decays $b \rightarrow s \gamma$ and $b \rightarrow d \gamma$, as well as their leptonic counterparts is possible which is more suitable for the experimental settings. It provides a stringent test, if the CKM matrix is indeed the only source of CP violation. Using U-spin, which is the $S U(2)$ subgroup of flavour $S U(3)$ relating the $s$ and the $d$ quark and which is already a wellknown tool in the context of nonleptonic decays [30,31], one derives relations between the $\mathrm{CP}$ asymmetries of the exclusive channels $B^{-} \rightarrow K^{*-} \gamma$ and $B^{-} \rightarrow \rho^{-} \gamma$ and of the inclusive channels $B \rightarrow X_{s} \gamma$ and $B \rightarrow X_{d} \gamma$. One should make use of the U-spin symmetry only with respect to the strong interactions. Moreover, within exclusive final states, the vector mesons like the U-spin doublet $\left(K^{*-}, \rho^{-}\right)$are favoured as final states because these have masses much larger than the (current-quark) masses of any of the light quarks. Thus one expects, for the ground-state vector mesons, the U-spin symmetry to be quite accurate in spite of the nondegeneracy of $m_{d}$ and $m_{s}$. Defining the rate asymmetries (not the normalized $\mathrm{CP}$ asymmetries) by

$$
\Delta \Gamma\left(B^{-} \rightarrow V^{-} \gamma\right)=\Gamma\left(B^{-} \rightarrow V^{-} \gamma\right)-\Gamma\left(B^{+} \rightarrow V^{+} \gamma\right)
$$

one arrives at the following relation [29]:

$$
\Delta \Gamma\left(B^{-} \rightarrow K^{*-} \gamma\right)+\Delta \Gamma\left(B^{-} \rightarrow \rho^{-} \gamma\right)=b_{e x c} \Delta_{e x c}
$$

where the right-hand side is written as a product of a relative U-spin breaking $b_{\text {exc }}$ and a typical size $\Delta_{\text {exc }}$ of the $\mathrm{CP}$ violating rate difference. In order to give an estimate of the right-hand side, one can use the model result from [23] for $\Delta_{e x c}$,

$$
\Delta_{\text {exc }}=2.5 \times 10^{-7} \Gamma_{B} .
$$

The relative breaking $b_{\text {exc }}$ of U-spin can be estimated, e.g. from spectroscopy. This leads us to

$$
\left|b_{e x c}\right|=\frac{M_{K^{*}}-m_{\rho}}{\frac{1}{2}\left(M_{K^{*}}+m_{\rho}\right)}=14 \% .
$$

Certainly, other estimates are also possible, such as a comparison of $f_{\rho}$ and $f_{K^{*}}$. In this case one finds a very small U-spin breaking. Using the more conservative value for $b_{\text {exc }}$, which is also compatible with sum rule calculations of form factors (see [32]), one arrives at the standard-model prediction for the difference of branching ratios

$$
\left|\Delta \mathcal{B}\left(B^{-} \rightarrow K^{*-} \gamma\right)+\Delta \mathcal{B}\left(B^{-} \rightarrow \rho^{-} \gamma\right)\right| \sim 4 \times 10^{-8}
$$


Note that the right-hand side is model-dependent.

Quite recently, the U-spin breaking effects were also estimated in the QCD factorization approach [33]. Within this approach, it was shown that the U-spin breaking effect essentially scales with the differences of the two form factors, $\left(F_{K^{*}}-F_{\rho}\right)$. Using the formfactors from the QCD sum rule calculation in [34] and maximizing the CP asymmetries by a specific choice of the CKM angle $\gamma$, the authors of [33] obtain

$$
\Delta \mathcal{B}\left(B^{-} \rightarrow K^{*-} \gamma\right)+\Delta \mathcal{B}\left(B^{-} \rightarrow \rho^{-} \gamma\right) \sim-3 \times 10^{-7}
$$

while for the separate asymmetries they obtain:

$$
\Delta \mathcal{B}\left(B \rightarrow K^{*} \gamma\right)=-7 \times 10^{-7}, \quad \Delta \mathcal{B}(B \rightarrow \rho \gamma)=4 \times 10^{-7}
$$

This calculation explicitly shows the limitations of the relation (9) as a SM test.

The issue is much more attractive in the inclusive modes. Due to the $1 / m_{b}$ expansion for the inclusive process, the leading contribution is the free $b$-quark decay. In particular, there is no sensitivity to the spectator quark and thus one arrives at the following relation for the CP rate asymmetries [29]:

$$
\Delta \Gamma\left(B \rightarrow X_{s} \gamma\right)+\Delta \Gamma\left(B \rightarrow X_{d} \gamma\right)=b_{i n c} \Delta_{i n c}
$$

In this framework one relies on parton-hadron duality; so one can actually compute the breaking of U-spin by keeping a nonvanishing strange quark mass. The typical size of $b_{\text {inc }}$ can be roughly estimated to be of the order of $\left|b_{\text {inc }}\right| \sim m_{s}^{2} / m_{b}^{2} \sim 5 \times 10^{-4} ;\left|\Delta_{\text {inc }}\right|$ is again the average of the moduli of the two $\mathrm{CP}$ rate asymmetries. These have been calculated (for vanishing strange quark mass), e.g. in [18] and thus one arrives at

$$
\left|\Delta \mathcal{B}\left(B \rightarrow X_{s} \gamma\right)+\Delta \mathcal{B}\left(B \rightarrow X_{d} \gamma\right)\right| \sim 1 \times 10^{-9}
$$

Any measured value in significant deviation of (16) would be an indication of new sources of $\mathrm{CP}$ violation. Although only an estimate is given here, it should again be stressed that in the inclusive mode the right-hand side in (16) can be computed in a model-independent way with the help of the heavy mass expansion.

Going beyond leading order in the $1 / m_{b}$ expansion the first subleading corrections are of order $1 / m_{b}^{2}$ only. The $1 / m_{b}^{2}$ corrections are induced by the imaginary part of the forward scattering amplitude $T(q)$ :

$$
T(q)=i \int d^{4} x<B\left|T O_{7}^{+}(x) O_{7}(0)\right| B>\exp (\text { iqx })
$$

where only the magnetic operator $O_{7}$ is taken into account. Using the operator product expansion for $T O_{7}^{+}(x) O_{7}(0)$ and heavy quark effective theory methods, the decay width $\Gamma\left(B \rightarrow X_{s} \gamma\right)$ reads $[38,39]$ (modulo higher terms in the $1 / m_{b}$ expansion):

$$
\begin{aligned}
\Gamma_{B \rightarrow X_{s} \gamma}^{\left(O_{7}, O_{7}\right)} & =\frac{\alpha G_{F}^{2} m_{b}^{5}}{32 \pi^{4}}\left|V_{t b} V_{t s}\right|^{2} C_{7}^{2}\left(m_{b}\right)\left(1+\frac{\delta^{N P}}{m_{b}^{2}}\right) \\
\delta^{N P} & =\frac{1}{2} \lambda_{1}-\frac{9}{2} \lambda_{2}
\end{aligned}
$$




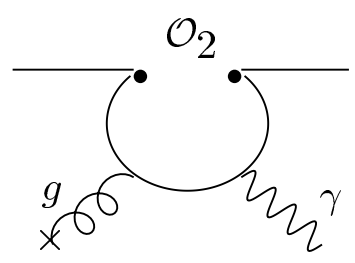

(a)

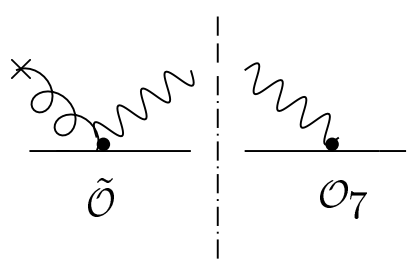

(b)

FIGURE 2. a) Feynman diagram from which the operator Õ arises. b) Relevant cut-diagram for the $\left(\mathrm{O}_{2}, \mathrm{O}_{7}\right)$-interference.

where $\lambda_{1}$ and $\lambda_{2}$ are the parameters for kinetic energy and the chromomagnetic energy. Using $\lambda_{1}=-0.5 \mathrm{GeV}^{2}$ and $\lambda_{2}=0.12 \mathrm{GeV}^{2}$, one gets $\delta^{N P} \simeq-4 \%$. Thus, the contributions are small and cancel in the sum of the rate asymmetries - in the limit of U-spin symmetry. The U-spin breaking effects in this contribution also induce an overall factor $m_{s}^{2} / m_{b}^{2}$ in addition - as one can read off from the explicit results for the $B \rightarrow X_{s} l^{+} l^{-}$case [35].

There are also nonperturbative corrections which scale with $1 / m_{c}^{2}$ [36]. which are induced by the interference of the mangnetic $O_{7}$ and the four-quark operator $O_{2}$. This effect is generated by the diagram in Fig. 2a (and by the one, not shown, where the gluon and the photon are interchanged); $g$ is a soft gluon interacting with the charm quarks in the loop. Up to a characteristic Lorentz structure, this loop is given by the integral

$$
\int_{0}^{1} d x \int_{0}^{1-x} d y \frac{x y}{m_{c}^{2}-k_{g}^{2} x(1-x)-2 x y k_{g} k_{\gamma}} .
$$

As the gluon is soft, i.e. $k_{g}^{2}, k_{g} k_{\gamma} \approx \Lambda^{Q C D} m_{b} / 2 \ll m_{c}^{2}$, the integral can be expanded in $k_{g}$. The (formally) leading operator, denoted by $\tilde{O}$, is

$$
\tilde{O}=\frac{G_{F}}{\sqrt{2}} V_{c b} V_{c s}^{*} C_{2} \frac{e Q_{c}}{48 \pi^{2} m_{c}^{2}} \bar{s} \gamma_{\mu}\left(1-\gamma_{5}\right) g_{s} G_{v \lambda} b \varepsilon^{\mu v \rho \sigma} \partial^{\lambda} F_{\rho \sigma} .
$$

Then working out the cut diagram shown in Fig. 2b, one obtains the nonperturbative contribution $\Gamma_{B \rightarrow X_{s} \gamma}^{\left(\tilde{O}, O_{7}\right)}$ to the decay width, which is due to the $\left(O_{2}, O_{7}\right)$ interference. Normalizing this contribution by the LL partonic width, one obtains

$$
\frac{\Gamma_{B \rightarrow X_{s} \gamma}^{\left(\tilde{O}, O_{Y}\right)}}{\Gamma_{b \rightarrow s \gamma}^{L L}}=-\frac{1}{9} \frac{C_{2}}{C_{7}} \frac{\lambda_{2}}{m_{c}^{2}} \simeq+0.03 .
$$

This result corresponds to the leading term in an expansion in the parameter $t=k_{g} k_{\gamma} / 2 m_{c}^{2}$. The expansion parameter is approximately $m_{b} \Lambda_{Q C D} / 2 m_{c}^{2} \approx 0.3$ (rather than $\Lambda_{Q C D}^{2} / m_{c}^{2}$ ) and it is not a priori clear whether formally higher order terms in the $m_{c}$ expansion are numerically suppressed. However, the explicit expansion of the complete vertex function, corresponding to Fig. 2a, shows that higher order terms are indeed suppressed, because the corresponding coefficients are small (see i.e. [37]). 
Moreover, the operator $\tilde{O}$ does not contain any information on the strange mass, thus, also in these contributions one finds the same overall suppression factor from the U-spin breaking.

The corresponding long-distance contributions from up-quark loops are CKM suppressed in the $B \rightarrow X_{s} \gamma$ case, but this does not hold in the $B \rightarrow X_{d} \gamma$ case. Naively, one could expect that the corresponding contributions from up-quark loops scale with $1 / m_{u}^{2}$. However, following the approach of [37], one easily shows that the general vertex function cannot be expanded in the parameter $t$ in that case. However, the expansion in inverse powers of $t$ is reasonable. The leading term in this expansion scales like $t^{-1} \sim m_{u}^{2} / k_{g} k_{\gamma}$ and therefore cancels the factor $1 / m_{u}^{2}$ in the prefactor (see the analogous $1 / m_{c}^{2}$ factor in (20)) and one gets a suppression factor $\left(\Lambda_{Q C D}^{2} / m_{u}^{2}\right) \cdot\left(m_{u}^{2} / k_{g} k_{\gamma}\right)$ [37]. Thus, although the expansion in inverse powers in $t$ induces nonlocal operators, one explicitly finds that the leading term scales with $\Lambda_{Q C D} / m_{b}{ }^{1}$.

This argument improves the discussion in [29] where the argument was given that vector dominance calculations in [40] show that the long-distance contributions from the up-quark loops to the decay rates are found to be rather small. However, that argument in [29] does not allow any statement about the effects of the U-spin breaking in constrast to the one given here.

Summing up, the analysis shows that the known nonperturbative contributions to (16) are under control and small and that this prediction provides a clean SM test, if generic new CP phases are present or not. Any significant deviation from the estimate (16) would be a strong hint to non-CKM contributions to $\mathrm{CP}$ violation.

Finally, we emphasize that an analogous prediction for the leptonic inclusive $B \rightarrow$ $X_{s / d} \gamma$ modes is also possible taking into account some specific cuts on the invariant dilepton spectrum.

\section{ACKNOWLEDGEMENTS}

We are grateful to Gerhard Buchalla for discussions and to Gino Isidori for a careful reading of the manuscript.

\section{REFERENCES}

1. T. Hurth, hep-ph/0106050.

2. CLEO Collaboration, M. S. Alam et al., Phys. Rev. Lett. 74, 2885 (1995).

3. CLEO Collaboration, S. Ahmed et al., hep-ex/9908022.

4. CLEO Collaboration, S. Chen et al., hep-ex/0108032.

5. ALEPH Collaboration, R. Barate et al., Phys. Lett. B 429, 169 (1998).

6. BELLE Collaboration, Y. Ushiroda, hep-ex/0104045.

7. New results presented by H. Tajima for the BELLE Collaboration and by D. Cassel for the CLEO Collaboration can be found at the homepage of the XX International Symposium on Lepton and Photon

\footnotetext{
${ }^{1}$ Also in the exclusive case an explicit analysis within the QCD factorization approach leads to this suppression factor [33].
} 
Interactions at High Energies 23-28 July 2001, Rome Italy: http://www.lp01.infn.it/.

8. A. Ali, C. Greub, Zeit. f. Phys. C60, 433 (1993); N. Pott, Phys. Rev. D54, 938 (1996); C. Greub, T. Hurth, D. Wyler, Phys. Lett. B380, 385 (1996); Phys. Rev. D54 3350 (1996); K. Adel, Y.P. Yao, Phys. Rev. D49 4945 (1994); C. Greub, T. Hurth, Phys. Rev. D56 2934 (1997); K. Chetyrkin, M. Misiak, M. Münz, Phys. Lett. B400 206 (1997).

9. G. Degrassi, P. Gambino, G. F. Giudice, JHEP0012, 009 (2000); M. Carena, D. Garcia, U. Nierste, C. E. Wagner, Phys. Lett. B 499, 141 (2001); W. de Boer, M. Huber, A. V. Gladyshev, D. I. Kazakov, hep-ph/0102163.

10. F. Gabbiani, E. Gabrielli, A. Masiero, L. Silvestrini, Nucl. Phys. B 477, 321 (1996); F. Borzumati, C. Greub, T. Hurth, D. Wyler, Phys. Rev. D 62, 075005 (2000); Nucl. Phys. Proc. Suppl. 86, 503 (2000)

11. T. Besmer, C. Greub and T. Hurth, Nucl. Phys. B 609, 359 (2001).

12. C. Greub and T. Hurth, Nucl. Phys. Proc. Suppl. 74, 247 (1999) [hep-ph/9809468].

13. P. Gambino and M. Misiak, hep-ph/0104034.

14. C. Greub and T. Hurth, hep-ph/9608449. Talk given at DPF 96 Meeting, Minneapolis, MN, 10-15 Aug 1996, in *Minneapolis 1996, Particles and fields, vol. 2*810-814.

15. M. Ciuchini, G. Degrassi, P. Gambino and G.F. Giudice, Nucl. Phys. B527, 21 (1998).

16. A.L. Kagan and M. Neubert, Eur. Phys. J. C7, 5 (1999).

17. T. Hurth et al., J. Phys. G G27, 1277 (2001).

18. A. Ali, H. Asatrian and C. Greub, Phys. Lett. B429, 87 (1998).

19. A. L. Kagan and M. Neubert, Phys. Rev. D 58, 094012 (1998).

20. K. Kiers, A. Soni and G. Wu, Phys. Rev. D 62, 116004 (2000).

21. F. Caravaglios, F. Parodi, P. Roudeau and A. Stocchi, hep-ph/0002171.

22. A. Ali and G. Hiller, Eur. Phys. J. C 8, 619 (1999).

23. C. Greub, H. Simma and D. Wyler, Nucl. Phys. B 434, 39 (1995) [Erratum-ibid. B 444, 447 (1995)].

24. T. E. Coan et al. [CLEO Collaboration], Phys.Rev.Lett. 86, 5661 (2001).

25. T. Goto, Y. Y. Keum, T. Nihei, Y. Okada and Y. Shimizu, Phys. Lett. B 460, 333 (1999).

26. M. Aoki, G. Cho and N. Oshimo, Nucl. Phys. B 554, 50 (1999); C. Chua, X. He and W. Hou,Phys. Rev. D 60, 014003 (1999); Y. G. Kim, P. Ko and J. S. Lee, Nucl. Phys. B 544, 64 (1999); S. Baek and P. Ko, Phys. Rev. Lett. 83, 488 (1999); L. Giusti, A. Romanino and A. Strumia, Nucl. Phys. B 550, 3 (1999); E. J. Chun, K. Hwang and J. S. Lee, Phys. Rev. D 62, 076006 (2000); D. Bailin and S. Khalil, Phys. Rev. Lett. 86, 4227 (2001).

27. H. H. Asatrian and H. M. Asatrian, Phys. Lett. B 460, 148 (1999); H. H. Asatryan, H. M. Asatrian, G. K. Yeghiyan and G. K. Savvidy, hep-ph/0012085.

28. A. G. Akeroyd, Y. Y. Keum and S. Recksiegel, Phys. Lett. B 507, 252 (2001).

29. T. Hurth and T. Mannel, Phys. Lett. B 511, 196 (2001).

30. R. Fleischer, Phys. Lett. B 459, 306 (1999).

31. M. Gronau and J. L. Rosner, Phys. Lett. B 500, 247 (2001); M. Gronau, Phys. Lett. B 492, 297 (2000).

32. A. Ali, V. M. Braun and H. Simma, Z. Phys. C 63, 437 (1994).

33. S. W. Bosch and G. Buchalla, hep-ph/0106081.

34. P. Ball and V. M. Braun, Phys. Rev. D 58, 094016 (1998).

35. A. Ali and G. Hiller, Eur. Phys. J. C 8, 619 (1999).

36. M.B. Voloshin, Phys. Lett. B397, 275 (1997); A. Khodjamirian, R. Ruckl, G. Stoll and D. Wyler, Phys. Lett. B402, 167 (1997); Z. Ligeti, L. Randall and M.B. Wise, Phys. Lett. B402, 178 (1997); A.K. Grant, A.G. Morgan, S. Nussinov and R.D. Peccei, Phys. Rev. D56, 3151 (1997).

37. G. Buchalla, G. Isidori and S. J. Rey, Nucl. Phys. B 511, 594 (1998).

38. A. F. Falk, M. Luke and M. J. Savage, Phys. Rev. D 49, 3367 (1994).

39. A. Ali, G. Hiller, L. T. Handoko and T. Morozumi, Phys. Rev. D 55, 4105 (1997).

40. G. Ricciardi, Phys. Lett. B 355, 313 (1995); N. G. Deshpande, X. He and J. Trampetic, Phys. Lett. B 367, 362 (1996). 